\title{
Performance of the Alere i RSV assay for point-of-care detection of respiratory syncytial virus in children
}

\author{
Sarah Valerie Schnee ${ }^{1,2,3+}$, Johannes Pfeil ${ }^{1 * \dagger} \mathbb{D}$, Clara Marlene Ihling ${ }^{2,3}$, Julia Tabatabai ${ }^{1,2,3}$ and Paul Schnitzler ${ }^{3}$
}

\begin{abstract}
Background: Respiratory syncytial virus (RSV) is the most important cause of severe acute respiratory tract infection in young children. Alere i RSV is a novel molecular rapid test which identifies respiratory syncytial virus in less than 13 min.

Methods: We evaluated the clinical performance of the Alere i RSV assay in a pediatric point-of-care setting during winter season 2016 / 2017. Test results from 518 nasopharyngeal swab samples were compared to a real-time reverse transcription PCR reference standard.

Results: The overall sensitivity and specificity of the Alere i RSV test assay was 93\% (Cl $\left.{ }^{95} 89 \%-96 \%\right)$ and $96 \%(C)^{95}$ $93 \%-98 \%)$, respectively. Alere i RSV performed well in children of all age groups. An optimal sensitivity of $98 \%$ $\left(\mathrm{Cl}^{95} 94 \%-100 \%\right)$ and specificity of 96\% (Cl ${ }^{95} 90 \%$ - 99\%) was obtained in children < 6 months. In children $\geq 2$ years, sensitivity and specificity remained at $87 \%\left(\mathrm{Cl}^{95} 73 \%-96 \%\right)$ and $98 \%\left(\mathrm{Cl}^{95} 92 \%-100 \%\right)$, respectively. False negative Alere i RSV test results mostly occurred in samples with low viral load (mean CT value 31.1; $\mathrm{Cl}^{95} 29.6-32$. 6). The Alere i RSV assay is easy to use and can be operated after minimal initial training. Test results are available within $13 \mathrm{~min}$, with most RSV positive samples being identified after approximately 5 min.
\end{abstract}

Conclusion: The Alere i RSV assay has the potential to facilitate the detection of RSV in pediatric point-of-care settings.

\section{Background}

Respiratory Syncytial Virus (RSV) is the most important cause of acute respiratory tract infection (aRTI) in neonates and young children worldwide [1]. RSV is a frequent cause of hospitalization in young children and leads to significant morbidity in premature neonates and children with chronic lung or congenital heart disease [2-4].

The clinical diagnosis of RSV is hampered by the mostly unspecific symptoms of RSV infection. Early recognition of RSV infection is useful to optimize care management, minimize unnecessary antibiotic use [5] and provide targeted infection control for children hospitalized with RSV infection [6]. In addition, specific

\footnotetext{
* Correspondence: Johannes.Pfeil@med.uni-heidelberg.de

${ }^{\dagger}$ Equal contributors

${ }^{1}$ Center for Childhood and Adolescent Medicine (General Pediatrics), University Hospital Heidelberg, Im Neuenheimer Feld 420, 69120 Heidelberg, Germany

Full list of author information is available at the end of the article
}

antiviral therapy for RSV infection is currently under early clinical evaluation [7], and early detection of RSV infection may become important for timely antiviral treatment in severely sick children.

Pediatricians therefore often apply rapid point-of-care RSV test assays. The major limitation of point-of-care RSV testing is the low sensitivity of commercially available rapid antigen detection tests (RADT). RADT sensitivity is strongly dependent on viral load, and therefore performs best in young infants with classical symptoms of RSV bronchiolitis. In older children and adults with low viral load, the sensitivity is poor and RADT are not recommended in these age groups $[8,9]$.

Alere i RSV is a novel rapid molecular test assay which can identify RSV in less than $13 \mathrm{~min}$. In a previous analysis, we reported an Alere i RSV sensitivity and specificity of $100 \%\left(\mathrm{CI}_{95} \quad 89-100 \%\right)$ and $97 \%\left(\mathrm{CI}_{95} 89 \%-\right.$ $100 \%)$, respectively [10]. This first analysis focused on young infants, and testing was done under laboratory 
conditions which may not reflect the performance in point-of-care settings.

In the current study, we addressed these limitations and applied the Alere i RSV test assay in a pediatric point-of-care setting on a larger study population across different pediatric age groups. The objective of this analysis was to report an estimate of the Alere i RSV test performance in a pediatric point-of-care setting.

\section{Methods}

\section{Study cohort and sampling procedure}

Between November 2016 and March 2017, we prospectively collected 533 nasopharyngeal swabs (NPS) in the outpatient department of the Center of Childhood and Adolescent Medicine Heidelberg, Germany. Study inclusion criteria were I) age $<18$ years, II) clinical symptoms of an acute respiratory infection, and III) indication for hospitalization according to the clinical judgment of the attending physician. Patients with clinical symptoms of an acute respiratory infection included cases of upper respiratory tract infection (URTI), otitis media, croup, bronchiolitis, bronchitis and pneumonia.

Nasopharyngeal swabs were collected by local staff in $1 \mathrm{ml}$ viral transport media (VTM; MSwab; Copan, Brescia, Italy). $200 \mu \mathrm{l}$ of the VTM were directly used for point-of-care testing with the Alere i RSV assay. The remaining sample was transferred to the virology diagnostic laboratory, and stored in $200 \mu \mathrm{l}$ aliquots at $-80{ }^{\circ} \mathrm{C}$ until further analysis.

Attending pediatricians prospectively reported medical information on a standardized data sheet, including the duration of clinical symptoms, demographic and clinical data.

\section{Alere i RSV assay}

Alere i RSV test assays were applied in the pediatric outpatient department. The test procedure followed the Alere i RSV package insert [11]. In brief, $200 \mu \mathrm{l}$ of the respiratory sample was added to the sample receiver containing $2.5 \mathrm{ml}$ elution buffer. Two $100 \mu \mathrm{l}$ volumes were added to the test base with the provided transfer cartridge for isothermal amplification.

Alere i RSV assays were conducted by attending physicians or nurses working in the pediatric outpatient department. All operators were allowed to carry out the test assay only after an initial hands-on-training. Test results were printed and reported on the medical datasheet. Invalid test results were re-tested immediately.

\section{Reference standard (RT-PCR test procedures)}

The Alere i RSV assay was evaluated against a CEmarked real-time reverse transcriptase polymerase chain reaction assay (RT-PCR).
For RT-PCR analysis, RNA was extracted from $140 \mu \mathrm{l}$ respiratory specimen using the QIAamp ${ }^{\circ}$ viral RNA mini kit (Qiagen, Hilden, Germany) according to the manufacturer's protocol. Amplification and detection of viral RNA was performed by FTD respiratory pathogens 21 multiplex PCR (FTD 21, Fast-track diagnostics Ltd., Sliema, Malta) on a LightCycler 480 instrument II (Roche, Mannheim, Germany). The FTD 21 assay can detect the following pathogens: influenza $A, H 1 N 1$ or $B$, rhinovirus, respiratory syncytial virus, bocavirus, adenovirus, parainfluenza $1,2,3$ or 4 , coronavirus NL63, 229E, OC43 or HKU1, parechovirus, enterovirus, human metapneumovirus A/B and Mycoplasma pneumoniae.

FTD 21 results that did not correspond to the Alere i test assay were verified by a second RT-PCR assay. For this purpose, RNA was extracted from an independent sample aliquot and analyzed by altona RealStar PCR (altona RealStar RSV RT-PCR, altona Diagnostics, Hamburg, Germany). To preclude possible discrepancies between the two RT-PCR methods, samples with different results in monoplex and multiplex PCR were again tested by the FTD 21 assay (Additional file 1: Figure S3).

Both the FTD 21 and the altona RealStar assay were initially evaluated using defined RSV A and RSV B positive and negative samples from patients, from the German RSV reference laboratory and from the official German proficiency testing panel. Results with a cycle threshold $\left(C_{T}\right)$ value of $<35$ were considered positive.

For sub-typing of RSV positive samples, Sanger sequencing targeting the second hyper-variable region of the $G$ gene was performed using primer pairs as previously described [12]. Resulting sequences were assembled and edited using the SEQMAN II software of the Lasergene package (DNAstar, Madison, WI) and allocated to subtype RSV A or RSV B using the Basic Local Alignment Search Tool (BLAST; http://blast.ncbi.nlm.nih.gov).

\section{Statistical analysis and data reporting}

Statistical analyses were conducted using Stata/IC13.0 (StataCorp. LP, College Station, TX, USA). MannWhitney-U-test was applied to compare $C_{T}$ values in samples with true positive versus false negative Alere $\mathrm{i}$ RSV result. The Alere i RSV sensitivity in RSV A versus RSV B positive samples was compared using the $\chi^{2}$-Test. $P$ values $<0.05$ were considered statistically significant. Data reporting was done according to STARD 2015 recommendations [13].

\section{Results}

From November 2016 to March 2017, 533 NPS were collected from 499 children presenting with symptoms of acute respiratory tract infection. Fifteen samples were excluded from the final analysis due to unavailability of sample aliquots for RT-PCR testing $(n=7)$, duplicate 


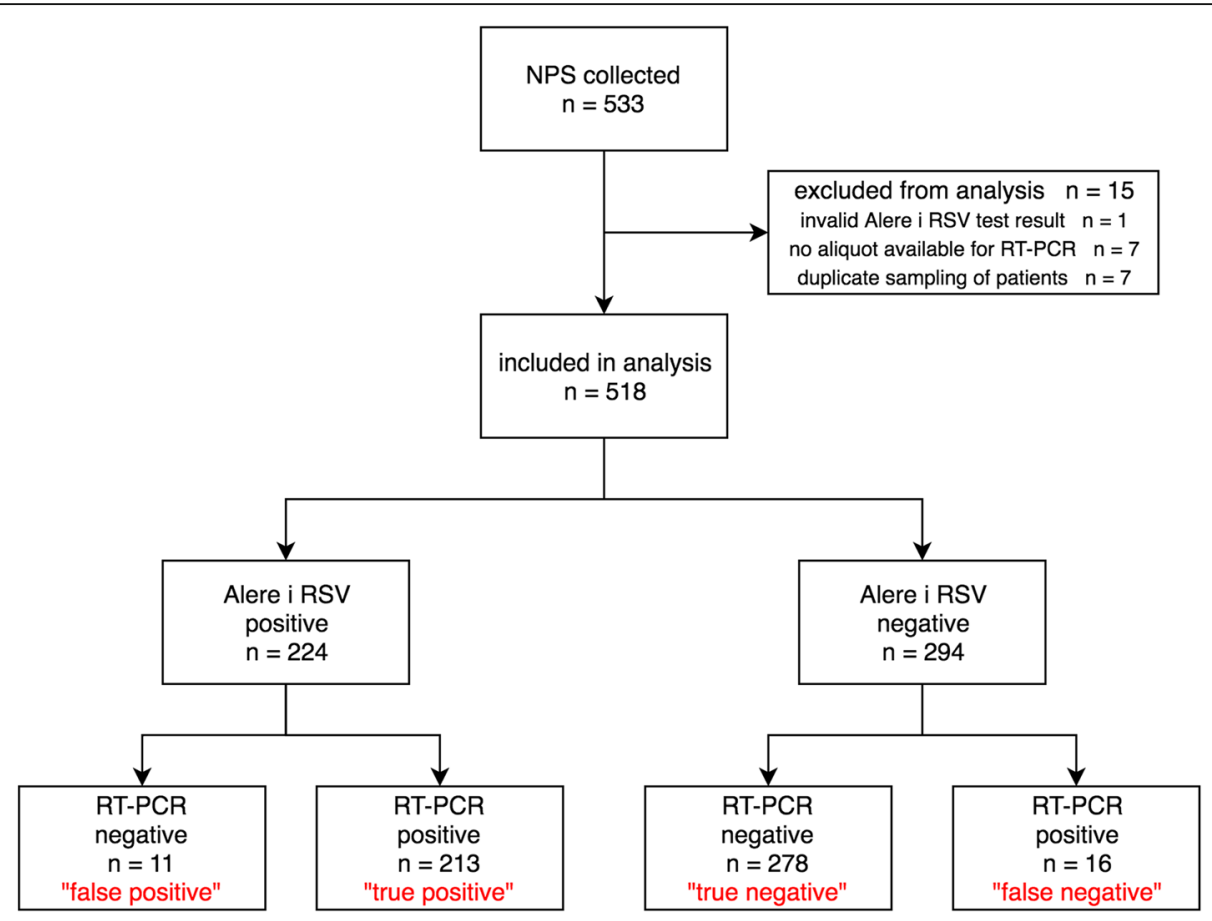

Fig. 1 Study Flow Chart

Table 1 Baseline demographic and clinical characteristics of 518 study participants

\begin{tabular}{lll}
\hline $\mathrm{N}=518$ & $\mathrm{n}$ & $\%$ of total \\
\hline gender & 292 & $57 \%$ \\
male & 226 & $43 \%$ \\
female & & \\
age & 212 & $41 \%$ \\
$0-5$ months & 76 & $15 \%$ \\
$6-11$ months & 107 & $21 \%$ \\
$12-23$ months & 123 & $24 \%$ \\
$\geq 2$ years & & \\
duration of clinical symptoms on admission & 276 & $53 \%$ \\
$\leq 3$ days & 218 & $42 \%$ \\
$>3$ days & 24 & $5 \%$ \\
unknown & & \\
admission diagnosis & 81 & $16 \%$ \\
URTI & 257 & $49 \%$ \\
LRTI & 180 & $35 \%$ \\
non-respiratory &
\end{tabular}

We grouped the admission diagnoses in cases of URTI $(n=81$, including URTI, otitis media and croup), LRTI ( $n=257$, including bronchiolitis, bronchitis and pneumonia) and non-respiratory admission diagnoses $(n=180)$. The latter includes children admitted for non-respiratory reasons (e.g. febrile convulsion, diarrhea) with concomitant acute RTI sampling of patients during one hospital stay $(n=7)$, or missed re-testing of an initially invalid Alere i RSV test result $(n=1)$.

Five hundred eighteen samples (97\% of 533 collected NPS) from 492 children were included in the final analysis (Fig. 1). Demographic and clinical characteristics of the study participants are summarized in Table 1.

Alere i RSV was positive in 43\% (224/518) and negative in $57 \%(294 / 518)$. In comparison to the RTPCR reference standard, the Alere i RSV test result was true positive in 213 and true negative in 278 samples, respectively. False positive test results were reported in 11 patients, and 16 patients were identified with false negative Alere i RSV test outcome (Table 2). The overall Alere i RSV test sensitivity and specificity was 93\% $\left(\mathrm{CI}_{95}\right.$ $89 \%-96 \%)$ and $96 \%\left(\mathrm{CI}_{95} 93 \%-98 \%\right)$, respectively.

The mean FTD $21 C_{T}$ value of true positive samples was $17.7\left(\mathrm{CI}_{95}\right.$ 17.1-18.3; range 11.7-34.6). NPS with false negative Alere i RSV result had a significantly higher mean $C_{T}$ value of $31.1\left(\mathrm{CI}_{95}\right.$ 29.6-32.6; range 25.4-34.5, $P<0$ 0.001; Mann-Whitney-U-test).

Table 2 Cross tabulation of Alere i RSV test results by the RT-PCR result

\begin{tabular}{llll}
\hline & Alere i RSV positive & Alere i RSV negative & total \\
\hline RT-PCR positive & 213 & 16 & 229 \\
RT-PCR negative & 11 & 278 & 289 \\
total & 224 & 294 & 518 \\
\hline
\end{tabular}


Table 3 Alere i RSV test sensitivity and specificity by admission diagnosis and age group

\begin{tabular}{|c|c|c|c|c|c|c|c|c|}
\hline & \multicolumn{2}{|c|}{ Alere i RSV test result } & \multirow[t]{2}{*}{ total } & \multirow{2}{*}{$\begin{array}{l}\text { sensitivity in } \\
\%\left(\mathrm{Cl}_{95}\right)\end{array}$} & \multicolumn{2}{|c|}{ Alere i RSV test result } & \multirow[t]{2}{*}{ total } & \multirow{2}{*}{$\begin{array}{l}\text { specificity } \\
\text { in \% }\left(\mathrm{Cl}_{95}\right)\end{array}$} \\
\hline & true positive & $\overline{\text { false negative }}$ & & & true negative & $\overline{\text { false positive }}$ & & \\
\hline & 213 & 16 & 229 & $93(89-96)$ & 278 & 11 & 289 & $96(93-98)$ \\
\hline \multicolumn{9}{|l|}{ admission diagnosis } \\
\hline URTI & 21 & 0 & 21 & $100(84-100)$ & 57 & 3 & 60 & 95 (86-99) \\
\hline LRTI & 159 & 6 & 165 & 96 (92-99) & 89 & 3 & 92 & 97 (91-99) \\
\hline non-respiratory & 33 & 10 & 43 & $77(61-88)$ & 133 & 5 & 138 & 96 (92-99) \\
\hline \multicolumn{9}{|l|}{ age group } \\
\hline $0-5$ months & 112 & 2 & 114 & $98(94-100)$ & 94 & 4 & 98 & $96(90-99)$ \\
\hline 6-11 months & 33 & 2 & 35 & $94(81-99)$ & 40 & 1 & 41 & $98(87-100)$ \\
\hline $12-23$ months & 34 & 7 & 41 & $83(68-93)$ & 62 & 4 & 66 & $94(85-98)$ \\
\hline$\geq 2$ years & 34 & 5 & 39 & 87 (73-96) & 83 & 2 & 85 & $98(92-100)$ \\
\hline
\end{tabular}

RSV A infection was detected in 65\% (149 / 229) and RSV B in 32\% (74 / 229) of RSV positive NPS. In two samples, both RSV A and B were identified. In 4 cases, no subtype could be determined. The sensitivity of the Alere i RSV assay was 93\% $\left(\mathrm{CI}_{95} 87 \%\right.$ - 96\%) in RSV A and $96 \%\left(\mathrm{CI}_{95} 89 \%\right.$ - 99\%) in RSV B positive samples. This difference was not statistically significant $(P=0.3$, $\chi^{2}$-Test).

From the clinical perspective, $C_{T}$ values were higher (and hence viral load lower) in respiratory specimens of older children and children admitted for non-respiratory reasons with concomitant respiratory tract infection (Additional file 1: Table S1). In consequence, the Alere i RSV sensitivity was 98\% $\left(\mathrm{CI}_{95} 94 \%-100 \%\right)$ in children $<6$ months, 94\% $\left(\mathrm{CI}_{95} 81 \%-99 \%\right)$ in children 611 months, $83 \%\left(\mathrm{CI}_{95} 68 \%-93 \%\right)$ in children $12-$ 23 months and $87 \%\left(\mathrm{CI}_{95} 73 \%-96 \%\right)$ in children above 2 years of age (Table 3). In children hospitalized for URTI or LRTI, the Alere i RSV test sensitivity was $100 \%$ $\left(\mathrm{CI}_{95}\right.$ 84-100\%) and 96\% ( $\mathrm{CI}_{95}$ 92-99\%), respectively. In children who were admitted for non-respiratory reasons, we found a sensitivity of $77 \%\left(\mathrm{CI}_{95} 61 \%\right.$ - 88\%) (Table 3 ). As illustrated in Additional file 1: Figure S2, the relatively poor sensitivity in this group resulted from a high proportion of samples with low viral load $\left(\mathrm{C}_{\mathrm{T}}\right.$ value $\left.>30\right)$, and coinfection with Influenza A, Parainfluenza, Rhinovirus or Coronavirus was detected in 5 / 10 cases.

We did not undertake comprehensive analytical specificity testing but note that the 289 NPS with negative RT-PCR result included samples tested positive for Influenza $A, H 1 N 1$ or $B(n=42)$, Rhinovirus $(n=99)$, Bocavirus $(n=23)$, Adenovirus $(n=18)$, Parainfluenza 1 , 2, 3 or $4(n=36)$, Coronavirus NL63, 229E, OC43 or HKU1 $(n=41)$, Parechovirus $(\mathrm{n}=3)$, Enterovirus $(\mathrm{n}=3)$, Human metapneumovirus $A / B(n=12)$ and Mycoplasma pneumoniae $(\mathrm{n}=3)$. In RSV positive specimens, observed coinfections included Rhinovirus $(n=38)$, Coronavirus $(n=25)$, Parainfluenza $(\mathrm{n}=12)$, Bocavirus $(\mathrm{n}=$
9) and Influenza $(n=8)$. In 65 cases, neither RSV nor any of the above-mentioned pathogens were detected.

In our point-of-care setting, positive test results were identified after a mean duration of $5.1 \mathrm{~min}\left(\mathrm{CI}_{95} 4.9-\right.$ $5.2 \mathrm{~min}$; range 4.7-10.0 $\mathrm{min}$ ). This included both sample pre-heating ( $3 \mathrm{~min}$ ) and the amplification reaction. After the first testing, invalid results were reported in 6\% (29/ 518 ). These NPS were directly retested, and valid (positive or negative) results were obtained in all cases.

\section{Discussion}

We found that the novel Alere i RSV assay has a sensitivity of $93 \%\left(\mathrm{CI}_{95} 89 \%-96 \%\right)$ and a specificity of $96 \%$ $\left(\mathrm{Cl}_{95} 93 \%-98 \%\right)$ in a pediatric point-of-care setting. The test is user-friendly and test results are obtained in less than $13 \mathrm{~min}$, with most positive test results being identified after approximately $5 \mathrm{~min}$.

No direct comparison of Alere i RSV versus RADT was done in our study. Based on published data, the expected RADT sensitivity in young children is approximately $80 \%$ [9]. In our study setting, we previously found a RADT sensitivity of $63 \%\left(\mathrm{CI}_{95} 55-72 \%\right)$ [14] and $55 \%$ $\left(\mathrm{CI}_{95} 45 \%\right.$ - 64\%) [15] over two different winter seasons. The Alere i RSV sensitivity is clearly superior to the expected RADT performance.

In children aged 24-35 months with lower viral load, the use of RADT is particularly limited with reported sensitivity of approximately $60 \%$ [16]. In patients $\geq 2$ years of age, we found an Alere i sensitivity and specificity of $87 \%$ and $98 \%$ in comparison to our RT-PCR reference standard, respectively. The Alere $\mathrm{i}$ assay is suitable for point-of-care detection of RSV in children across all age groups.

Other sensitive rapid nucleic acid amplification assays are available for early detection of RSV infection. These assays require a testing time of at least $30 \mathrm{~min}$ to more than $1 \mathrm{~h}[17,18]$. Alere i RSV test results are available 
within $13 \mathrm{~min}$, and positive test results are called out after a mean duration of approximately $5 \mathrm{~min}$.

A limitation of our study is the use of VTM for Alere $i$ RSV testing instead of directly inserting the swab to the Alere test base. Using VTM was required to establish the RT-PCR reference standard. This procedure is in accordance with the Alere i RSV package insert, but implies that samples are 1:5 diluted in comparison to directly inserting the swab. In our analysis, this could have provoked false negative results in samples with low viral load, and regular point-of-care users might prefer direct, non-diluted testing of swab specimens. Second, we compared Alere i RSV test results against a multiplex RTPCR reference standard. Only divergent results were further evaluated by monoplex RT-PCR. Multiplex RT-PCR is usually slightly less sensitive than monoplex RT-PCR in samples with low viral load. [19] In pediatric patients, viral loads are usually high and in fact, $95 \%$ of our RSV positive samples had a $C_{T}$ value $<30$. We therefore believe that multiplex RT-PCR is a rigorous reference standard in our study cohort, but acknowledge that applying a monoplex RT-PCR reference standard might have resulted in a slightly lower sensitivity of the Alere $i$ RSV assay.

In summary, we evaluated the novel Alere i RSV assay in a pediatric emergency setting against a RT-PCR reference standard. The Alere i RSV performed well in the point-of-care setting, and sensitive test results were obtained across all pediatric age groups within $13 \mathrm{~min}$. The assay requires a shorter test time than other currently available molecular test assays, and provides a significantly higher sensitivity than RADT assays.

\section{Conclusions}

The Alere i RSV assay performs well in the pediatric point-of-care setting. The assay is easy to use, and the high sensitivity and specificity of test results help pediatricians to act appropriately both in patients with and without RSV infection.

\section{Additional file}

Additional file 1: Supplementary Files (DOCX 206 kb)

\section{Abbreviations \\ altona RealStar PCR: altona RealStar RSV RT-PCR, altona Diagnostics, Hamburg, Germany; aRTI: acute respiratory tract infection; $C_{T}$ : cycle threshold; FTD 21: FTD respiratory pathogens 21 multiplex PCR; LRTI: lower respiratory tract infection; NPS: nasopharyngeal swabs; RADT: rapid antigen detection tests; RSV: Respiratory syncytial virus; RT-PCR: real-time reverse transcriptase polymerase chain reaction assay; URTI: upper respiratory tract infection; VTM: viral transport media}

\section{Acknowledgements}

We thank the physicians and nurses at the Center for Childhood and Adolescent Medicine for collecting respiratory samples and the technicians in the virology diagnostic laboratory for excellent technical assistance.

\section{Funding}

Alere i RSV and RT-PCR tests were partly provided by Alere free of charge. Alere had no influence on the study procedure and analysis of study results. S.V.S., C.I. and J.T. are financially supported by fellowships of the German Center for Infectious Diseases (DZIF). J.P. is the recipient of an HRCMM (Heidelberg Research Center for Molecular Medicine) Career Development Fellowship.

\section{Availability of data and materials}

All data generated or analysed during this study are included in this published article and its supplementary information files.

\section{Authors' contributions}

SVS, JP and CMI conducted study experiments. SVS and JP wrote the manuscript. All authors (SVS, JP, CMI, JT and PS) contributed to the interpretation of study results and approved the final version of this manuscript.

\section{Ethics approval and consent to participate}

This study was approved by the Ethical Research Board of the University Hospital Heidelberg, Germany (S-547/2015). All samples and medical information included in this study were obtained during routine medical care. Written consent for the analysis and publication of the data included in this study was obtained from all parents or guardians.

\section{Consent for publication}

Not applicable

\section{Competing interests}

Alere i RSV and RT-PCR test materials were partly provided by Alere free of charge.

\section{Publisher's Note}

Springer Nature remains neutral with regard to jurisdictional claims in published maps and institutional affiliations.

\section{Author details \\ ${ }^{1}$ Center for Childhood and Adolescent Medicine (General Pediatrics), University Hospital Heidelberg, Im Neuenheimer Feld 420, 69120 Heidelberg, Germany. ${ }^{2}$ German Center for Infection Research (DZIF), Heidelberg partner site, Heidelberg, Germany. ${ }^{3}$ Center for Infectious Diseases, Virology, University Hospital Heidelberg, Heidelberg, Germany.}

Received: 23 August 2017 Accepted: 26 November 2017

Published online: 13 December 2017

\section{References}

1. Nair H, Nokes DJ, Gessner BD, Dherani M, Madhi SA, Singleton RJ, O'Brien KL, Roca A, Wright PF, Bruce N, et al. Global burden of acute lower respiratory infections due to respiratory syncytial virus in young children: a systematic review and meta-analysis. Lancet. 2010;375:1545-55.

2. Bashir U, Nisar N, Arshad Y, Alam MM, Ashraf A, Sadia H, Kazi BM, Zaidi SS. Respiratory syncytial virus and influenza are the key viral pathogens in children $<2$ years hospitalized with bronchiolitis and pneumonia in Islamabad Pakistan. Arch Virol. 2017;162:763-73.

3. Iwane MK, Edwards KM, Szilagyi PG, Walker FJ, Griffin MR, Weinberg GA, Coulen C, Poehling KA, Shone LP, Balter S, et al. Population-based surveillance for hospitalizations associated with respiratory syncytial virus, influenza virus, and parainfluenza viruses among young children. Pediatrics. 2004;113:1758-64

4. Schanzer DL, Langley JM, Tam TW. Hospitalization attributable to influenza and other viral respiratory illnesses in Canadian children. Pediatr Infect Dis J. 2006:25:795-800.

5. Adcock PM, Stout GG, Hauck MA, Marshall GS. Effect of rapid viral diagnosis on the management of children hospitalized with lower respiratory tract infection. Pediatr Infect Dis J. 1997;16:842-6.

6. Madge P, Paton JY, McColl JH, Mackie PL. Prospective controlled study of four infection-control procedures to prevent nosocomial infection with respiratory syncytial virus. Lancet. 1992;340:1079-83.

7. Jorquera PA, Tripp RA. Respiratory syncytial virus: prospects for new and emerging therapeutics. Expert review of respiratory medicine. 2017; 
8. Casiano-Colon AE, Hulbert BB, Mayer TK, Walsh EE, Falsey AR. Lack of sensitivity of rapid antigen tests for the diagnosis of respiratory syncytial virus infection in adults. Journal of clinical virology : the official publication of the Pan American Society for Clinical Virology. 2003;28:169-74.

9. Chartrand C, Tremblay N, Renaud C, Papenburg J. Diagnostic accuracy of rapid antigen detection tests for respiratory Syncytial virus infection: systematic review and meta-analysis. J Clin Microbiol. 2015;53:3738-49.

10. Peters RM, Schnee SV, Tabatabai J, Schnitzler P, Pfeil J. Evaluation of Alere i RSV for rapid detection of respiratory syncytial virus in children hospitalized with acute respiratory tract infection. J Clin Microbiol. 2017;

11. Alere i RSV package insert. Available at [http://www.alere.com/en/home/ product-details/alere-i-rsv.html].

12. Peret $\mathrm{TC}$, Hall CB, Schnabel KC, Golub JA, Anderson LJ. Circulation patterns of genetically distinct group $a$ and $B$ strains of human respiratory syncytial virus in a community. The Journal of general virology. 1998;79(Pt 9):2221-9.

13. Bossuyt PM, Reitsma JB, Bruns DE, Gatsonis CA, Glasziou PP, Irwig L, Lijmer JG, Moher D, Rennie D, de Vet HC, et al. STARD 2015: an updated list of essential items for reporting diagnostic accuracy studies. BMJ. 2015;351:h5527.

14. Pfeil J, Tabatabai J, Sander A, Ries M, Grulich-Henn J, Schnitzler P. Screening for respiratory syncytial virus and isolation strategies in children hospitalized with acute respiratory tract infection. Medicine. 2014;93:e144.

15. Hoos J, Peters RM, Tabatabai J, Grulich-Henn J, Schnitzler P, Pfeil J. Reversetranscription loop-mediated isothermal amplification for rapid detection of respiratory syncytial virus directly from nasopharyngeal swabs. J Virol Methods. 2017;242:53-7.

16. Papenburg J, Buckeridge DL, De Serres G, Boivin G. Host and viral factors affecting clinical performance of a rapid diagnostic test for respiratory Syncytial virus in hospitalized children. J Pediatr. 2013;

17. Wahrenbrock MG, Matushek S, Boonlayangoor S, Tesic V, Beavis KG, Charnot-Katsikas A. Comparison of Cepheid Xpert flu/RSV XC and BioFire FilmArray for detection of influenza a, influenza B, and respiratory Syncytial virus. J Clin Microbiol. 2016;54:1902-3.

18. Salez N, Nougairede A, Ninove L, Zandotti C, de Lamballerie X, Charrel RN. Prospective and retrospective evaluation of the Cepheid Xpert(R) flu/RSV XC assay for rapid detection of influenza a, influenza B, and respiratory syncytial virus. Diagn Microbiol Infect Dis. 2015;81:256-8.

19. Sakthivel SK, Whitaker B, Lu X, Oliveira DB, Stockman L, Kamili S, Oberste MS, Erdman DD. Comparison of fast-track diagnostics respiratory pathogens multiplex real-time RT-PCR assay with in-house singleplex assays for comprehensive detection of human respiratory viruses. J Virol Methods. 2012:185:259-66.

\section{Submit your next manuscript to BioMed Central and we will help you at every step:}

- We accept pre-submission inquiries

- Our selector tool helps you to find the most relevant journal

- We provide round the clock customer support

- Convenient online submission

- Thorough peer review

- Inclusion in PubMed and all major indexing services

- Maximum visibility for your research

Submit your manuscript at www.biomedcentral.com/submit

) Biomed Central 